\title{
Studying the accretion geometry of EXO 2030+375 at luminosities close to the propeller regime
}

F. Fürst ${ }^{1}$, P. Kretschmar ${ }^{1}$, J. J. E. Kajava ${ }^{2,3,1}$, J. Alfonso-Garzón ${ }^{4}$, M. Kühnel ${ }^{5}$, C. Sanchez-Fernandez ${ }^{1}$, P. Blay ${ }^{6,7}$, C. A. Wilson-Hodge ${ }^{8}$, P. Jenke ${ }^{8}$, I. Kreykenbohm ${ }^{5}$, K. Pottschmidt ${ }^{9}, 10$, J. Wilms ${ }^{5}$, and R. E. Rothschild ${ }^{11}$

${ }^{1}$ European Space Astronomy Centre (ESAC), Science Operations Departement, 28692 Villanueva de la Cañada, Madrid, Spain e-mail: felix.fuerst@sciops.esa.int

2 Finnish Centre for Astronomy with ESO (FINCA), University of Turku, Väisäläntie 20, 21500 Piikkiö, Finland

3 Tuorla Observatory, University of Turku, Väisäläntie 20, 21500 Piikkiö, Finland

${ }^{4}$ Centro de Astrobiología - Departamento de Astrofísica (CSIC-INTA), Camino Bajo del Castillo s/n, Urb. Villafranca del Castillo, 28691 Villanueva de la Cañada, Madrid, Spain

5 Dr. Karl-Remeis-Sternwarte and ECAP, Sternwartstr. 7, 96049 Bamberg, Germany

${ }^{6}$ Instituto de Astrofísica de Canarias, 38200 Lalaguna, Tenerife, Spain

7 Nordic Optical Telescope, 38700 S/C de La Palma, La Palma, Spain

8 NASA Marshall Space Flight Center, Huntsville, AL 35812, USA

9 CRESST, Department of Physics, and Center for Space Science and Technology, UMBC, Baltimore, MD 21250, USA

10 NASA Goddard Space Flight Center, Greenbelt, MD 20771, USA

11 Center for Astrophysics and Space Sciences, University of California, San Diego, 9500 Gilman Dr, La Jolla, CA 92093-0424, USA

Received 5 April 2017 / Accepted 20 July 2017

\begin{abstract}
The Be X-ray binary EXO2030+375 was in an extended low-luminosity state during most of 2016. We observed this state with NuSTAR and Swift, supported by INTEGRAL observations and optical spectroscopy with the Nordic Optical Telescope (NOT). We present a comprehensive spectral and timing analysis of these data here to study the accretion geometry and investigate a possible onset of the propeller effect. The $\mathrm{H} \alpha$ data show that the circumstellar disk of the Be-star is still present. We measure equivalent widths similar to values found during more active phases in the past, indicating that the low-luminosity state is not simply triggered by a smaller Be disk. The NUSTAR data, taken at a $3-78 \mathrm{keV}$ luminosity of $\sim 6.8 \times 10^{35} \mathrm{erg} \mathrm{s}^{-1}$ (for a distance of $7.1 \mathrm{kpc}$ ), are nicely described by standard accreting pulsar models such as an absorbed power law with a high-energy cutoff. We find that pulsations are still clearly visible at these luminosities, indicating that accretion is continuing despite the very low mass transfer rate. In phaseresolved spectroscopy we find a peculiar variation of the photon index from $\sim 1.5$ to $\sim 2.5$ over only about $3 \%$ of the rotational period. This variation is similar to that observed with XMM-Newton at much higher luminosities. It may be connected to the accretion column passing through our line of sight. With Swift/XRT we observe luminosities as low as $10^{34} \mathrm{erg} \mathrm{s}^{-1}$ where the data quality did not allow us to search for pulsations, but the spectrum is much softer and well described by either a blackbody or soft power-law continuum. This softer spectrum might be due to the accretion being stopped by the propeller effect and we only observe the neutron star surface cooling.
\end{abstract}

Key words. X-rays: binaries - stars: neutron - accretion, accretion disks - magnetic fields

\section{Introduction}

Transient, accreting neutron stars in Be X-ray binaries can show luminosity variations over two to three orders of magnitude or more (e.g., Negueruela et al. 1998, and references therein). This variability allows us to investigate the $\mathrm{X}$-ray producing region in the accretion column over a wide variety of physical conditions, i.e., over regimes in which different interaction processes between the infalling material and the $\mathrm{X}$-ray radiation are dominant.

An open question is if and how accretion takes place at the lowest luminosities. Here, the strong magnetic field of the neutron star might inhibit further accretion, in what is known as the "propeller regime" (Illarionov \& Sunyaev 1975). In this regime, the magnetospheric radius becomes larger than the co-rotation radius, due to the reduced ram pressure of lower density material surrounding the neutron star. This effect inhibits accretion, leading to a drastically reduced accretion luminosity. Any observed residual flux might then originate from the hot neutron star surface or weak residual accretion at the poles or might be due to instabilities at the magneotsphere.

On the other hand, Tsygankov et al. (2017) have shown that for slow rotators a cold accretion disk could form, preventing the source from entering the propeller regime. Obtaining a better understanding of if and how accretion takes place at very low luminosities will help inform accretion modeling and allows us to study the magnetospheric interactions in greater detail. However, the intrinsic low fluxes make this regime difficult to observe.

One of the best studied Be X-ray binaries is EXO 2030+375, a neutron star in orbit with a B0 Ve star (Reig et al. 1998) at a distance of about $7.1 \mathrm{kpc}$ (Wilson et al. 2002). Since its discovery in 1985 (Coe et al. 1988; Parmar et al. 1989b) it has shown outbursts very regularly close to each periastron passage of its $45 \mathrm{~d}$ orbit, which are called Type I outbursts. They occur when 
the eccentric orbit of the neutron star brings it close enough to the circumstellar disk of its companion to trigger enhanced mass transfer. Type I outbursts are therefore significantly shorter than the orbital period, typically with durations between 10 and 20 days. In EXO 2030+375 over 150 Type I outbursts have been observed (Laplace et al. 2017).

In addition to the many Type I outbursts, EXO 2030+375 showed two much larger Type II outbursts in 1985 and 2006, that lasted for multiple orbital cycles. These events reach much higher luminosities and are likely due to increased activity of the Be-star and complex interaction between the neutron star and the circumstellar disk. These interactions lead to instabilities in which the mass transfer onto the neutron star is further increased (e.g., Okazaki \& Negueruela 2001). These spectacular outbursts were intensely monitored and led to insights about the high-luminosity accretion regime (Parmar et al. 1989a,b; Reynolds et al. 1993; Klochkov et al. 2007; Wilson et al. 2008). For example, EXO 2030+375 was the first pulsar in which a dramatic change in the pulse profile was observed as a function of luminosity, indicating a change in the emission pattern of the accretion column (Parmar et al. 1989b).

Ferrigno et al. (2016) studied phase-resolved XMM-Newton and Suzaku data of EXO 2030+375 during Type I outbursts in detail. They found a unique feature in the pulse profile, where the spectral hardness varies drastically over only about $2-3 \%$ of the rotational period. They interpret this feature as part of the accretion column passing through our line of sight towards the main $\mathrm{X}$-ray producing region, resulting in a higher absorption column and in a more reprocessed spectrum. Ferrigno et al. (2016) argue that the accretion column grows in size with increasing X-ray luminosity and therefore the duration of the feature should also increase. However, due to the limited phase-space resolution possible with Suzaku, they could not confirm this theory using a brighter Suzaku observation.

While Type I outbursts always occur close to periastron passage, their exact orbital phase has varied with time in EXO 2030+375. In particular, Wilson et al. (2002) observed that in late 1995 the outbursts occurred a few days before periastron, instead of after, but were overall fainter compared to previous outbursts. Around that time the source also switched to a long-term spin-down of its $42 \mathrm{~s}$ pulse period, the first such trend observed for a source that was still showing regular outbursts (Wilson et al. 2002).

Wilson et al. (2005) found that in 2003 EXO 2030+375 switched back to a spin-up trend and that the outbursts became brighter again, which led them to postulate a roughly $11-y r$ cycle of the activity of EXO 2030+375. By comparing the complete history of the pulse period and the outburst phase between 1985 and 2016, Laplace et al. (2017) also find an 11-yr periodicity; however, these authors also suggest that the true period might be about twice as long, i.e., 21 yr. On this period, giant outbursts alternate with a series of faint outbursts, explained by the alternating maxima of eccentricity and inclination of the Be-disk due to the Kozai-Lidov effect (Martin et al. 2014). In early 2015 the regularity of the outbursts started to break up again and an orbital phase shift was observed in 2016 July, which seems to confirm this 21-yr period (see also Laplace et al. 2016).

During the start of the faint phase in 2015 an outburst was observed only at every other periastron passage. Soon after, X-ray monitors like Swift/BAT, MAXI, and Fermi/GBM could not detect any activity (Fürst et al. 2016b). This period of quiescence provided the ideal opportunity to study EXO 2030+375 at the lowest possible luminosities to search for evidence of changes in the accretion geometry and for the onset of the propeller effect. We triggered a director's discretionary time target-ofopportunity observation with the Nuclear Spectroscopic Telescope Array $(N U S T A R)$ as well as a longer Swift observation campaign to follow several periastron passages.

As noted by Kretschmar et al. (2016) and Laplace et al. (2017) the outbursts of EXO 2030+375 picked up again by the end of 2016. Since then, the peak flux of the Type I outbursts occurs around orbital phase $\phi=0.0$, based on the ephemeris of Fit 2 by Wilson et al. (2008). This phase of maximum flux is significantly earlier in phase than the regular outbursts before 2015 , which occurred closer to $\phi=0.1$, i.e., about 4-5 days later (Kretschmar et al. 2017). At the time of writing, EXO 2030+375 appears to have entered a more regular outburst regime, with sequential outbursts from December 2016 to March 2017. All these outbursts also peak at the earlier phase, around $\phi=0.0$.

In this paper we present timing and spectral analysis of NuSTAR and Swift data, as well as an analysis of serendipitous observations with the International Gamma-Ray Astrophysics Laboratory (INTEGRAL) and optical spectroscopy with the Nordic Optical Telescope (NOT) taken during the quiescence period of 2016. In Sect. 2 we describe the data reduction. In Sect. 3 we perform spectral analysis of the NuSTAR observation. In Sect. 4 we put these NuSTAR results into context and describe the long-term flux and timing evolution. We discuss our results in Sect. 5 and present our conclusions in Sect. 6.

\section{Observations and data reduction}

All X-ray data reduction was performed with HEASOFT v6.19. Data analysis was performed with the Interactive Spectral Interpretation System (ISIS, Houck \& Denicola 2000) v1.6.2. All uncertainties are reported at the $90 \%$ confidence level for one parameter of interest unless otherwise noted.

\subsection{NUSTAR}

NuSTAR (Harrison et al. 2013) observed EXO 2030+375 starting on 2016 July 25, 08:36 UTC (ObsID 90201029002). We extracted the data using the standard software nupipeline v1.6 and CALDB v20160606. To increase the usable exposure time, we also extracted SCIENCE_SC data (mode 06), following the procedures as discussed by Walton et al. (2016) and Fürst et al. (2016a). This approach resulted in an exposure time of $51 \mathrm{ks}$ for each module.

The source spectra were extracted separately for each focal plane module and data mode, using a circular region with $40^{\prime \prime}$ radius centered on the source image in the respective detector. The region size was chosen to optimize the signal-to-noise ratio $(\mathrm{S} / \mathrm{N})$ at the highest energies: a larger region adds more background than source photons above $60 \mathrm{keV}$. Background spectra were extracted from a circular region with $120^{\prime \prime}$ radius on the same quadrant of the detector as the source, avoiding all visible stray light.

\subsection{Swift}

We extracted data from the X-ray Telescope (XRT, Burrows et al. 2005) from all 31 Swift (Gehrels et al. 2004) pointings between 2016 March and 2016 October using our custom scripts based on the standard XRT extraction method using xrtpipeline, xselect, and xrtmkarf. All observations were performed in photon-counting mode and have a typical exposure time of about $1 \mathrm{ks}$. We mostly used a circular source 
extraction region with a radius of $30^{\prime \prime}$; however, for the bright observations we used an annulus region with an inner radius of $15^{\prime \prime}$ and outer radius of $45^{\prime \prime}$ to avoid pile-up. The background was extracted from a circular region with $200^{\prime \prime}$ radius.

\subsection{INTEGRAL}

EXO 2030+375 was serendipitously observed by INTEGRAL (Winkler et al. 2003) during a series of public observations of Cyg X-1 scheduled for 2016 October 27-31 (Prop. ID 1220044). The INTEGRAL IBIS/ISGRI instrument (Lebrun et al. 2003) provided complementary hard X-ray data to the soft X-ray coverage of Swift. Given the moderate source brightness over this period, we filtered the available data set and selected only those pointings for which the source was detected within the $8.3 \times 8.0$ Fully Coded Field of View of IBIS/ISGRI. This selection resulted in a net exposure time of $194 \mathrm{ks}$. The IBIS/ISGRI data reduction was performed using the Off-line Scientific Analysis software (OSA; Courvoisier et al. 2003) v10.2, using the latest calibration files. Following standard reduction procedures the data were processed from the COR step to the SPE step.

\subsection{Nordic Optical Telescope}

The NOT is a $2.5 \mathrm{~m}$ optical telescope located on the Observatorio del Roque de los Muchachos, La Palma, Canarias. We took spectra with its ALFOSC spectrograph during the nights of 2016 June 14, July 31, August 21, and September 21. ALFOSC was equipped with a e 2 v CCD, with $2048 \times 2064$ pixel size. The instrumental setup included a $1^{\prime \prime}$ slit and a 485 lines per millimeter volume phase holographic grism, with a central wavelength of $7850 \AA$, a dispersion of $2.2 \AA$ per pixel, and a resolution of 770 , covering the wavelength range 5650-10 $150 \AA$. The data were reduced with IRAF (Tody 1993), applying standard procedures for long-slit spectroscopy.

\section{NuSTAR spectroscopy}

\subsection{Phase-averaged spectra}

To compare the EXO2030+375 low-luminosity spectrum to previous observations, we fit the phase-averaged NUSTAR data together with almost simultaneous Swift/XRT data (ObsID 00030799022) with typical phenomenological models often used to describe the X-ray spectra of these systems. We rebinned the NUSTAR data within ISIS to a S/N of 6 below $45 \mathrm{keV}$ and 3 above, while requiring a rebinning factor of at least 2 . This approach allows us to use the data between $3-70 \mathrm{keV}$. The XRT data were rebinned to a $\mathrm{S} / \mathrm{N}$ of 3 throughout, which provided useful data between $1-8 \mathrm{keV}$. The XRT data were taken a bit later in the outburst where the flux already started declining, hence showing a lower flux than the NUSTAR data, which we model via a multiplicative constant. However, the changes in spectral shape over this time range are negligible.

EXO 2030+375 is known to show complex absorption that is often fitted with a partially covering absorber (see, e.g., Naik et al. 2013; Ferrigno et al. 2016). We modeled the data using a global absorption column, $N_{\mathrm{H}, 1}$, and an additional partially covering column, $N_{\mathrm{H}, 2}$, with a covering fraction $f$. To describe the absorption we used an updated version of the tbabs model $^{1}$ by Wilms et al. (2000). We used the abundance provided

\footnotetext{
1 http://pulsar.sternwarte.uni-erlangen.de/wilms/ research/tbabs/
}

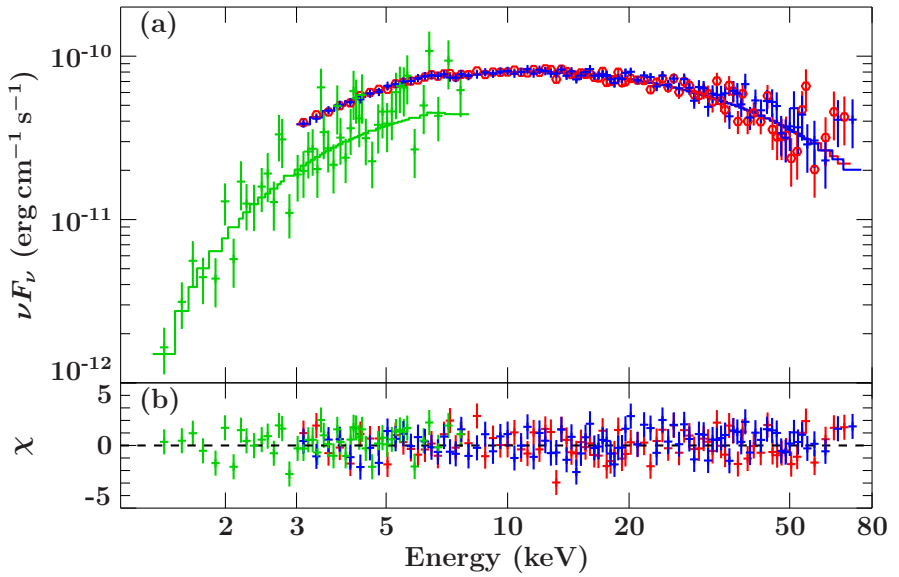

Fig. 1. Panel $a$ : Unfolded Swift/XRT (green), NuSTAR/FPMA (red), and $N u S T A R / F P M B$ (blue) phase-averaged spectra, together with the bestfit highecut model. Panel $b$ : Residual in terms of $\chi^{2}$ for the best-fit model. Data were strongly rebinned for clarity.

by these authors and the cross sections by Verner et al. (1996). In ISIS this model can be expressed as

$N_{\mathrm{H}, 1} \times\left[f N_{\mathrm{H}, 2}+(1-f)\right] \times$ continuum.

As continua we tried cutoffpl, highecut, NPEX (Mihara et al. 1998), and FDcut (Tanaka 1986). The results are listed in Table 1. A summary of the definitions of each of these models can be found in Müller et al. (2013). We find that neither the cutoffpl nor the FDcut provided a statistically acceptable fit to the data, while both highecut and NPEX resulted in very good and statistically equivalent fits. For the highecut model we also show the results without the partially covering absorber in Table 1, which is significantly worse, with an F-test false detection probability of $3.7 \times 10^{-5}$.

While we cannot statistically distinguish between the highecut and NPEX model, we will base the remaining discussion on the highecut model as Naik et al. (2013) found that it describes their data better. The highecut model was also successfully applied to EXO $2030+375$ by Wilson et al. (2008) and Klochkov et al. (2008), and therefore allows a direct comparison with their results. Our results are all qualitatively the same for the NPEX model. Figure 1 shows the XRT and NuSTAR spectrum, together with the best-fit highecut model.

We also tested a model consisting of a single absorbed highecut with an additional blackbody component at low energies for direct comparison with the results by Reig \& Coe (1999). This model results in the lowest $\chi_{\text {red }}^{2}$ value, but statistically it is not significantly better than the partially covered version.

Furthermore we searched for a neutral $\mathrm{Fe} \mathrm{K} \alpha$ line, modeled by a narrow Gaussian at $6.4 \mathrm{keV}$, that has often been observed in this source (e.g., Reig \& Coe 1999; Naik et al. 2013; Ferrigno et al. 2016). We do not find clear evidence of the presence of this line, with a $90 \%$ upper limit on the equivalent width around $25-30 \mathrm{eV}$, independent of the choice of the continuum model (Table 1).

\subsection{Phase-resolved spectroscopy}

Following the discovery of a peculiar feature in the phaseresolved data of XMM-Newton by Ferrigno et al. (2016), we extracted NUSTAR spectra in 100 phase-bins. We used a local 
Table 1. Best-fit model parameters for the phase-averaged fits.

\begin{tabular}{|c|c|c|c|c|c|c|}
\hline Parameter & HighE (no PC) & HighE (with PC) & HighE+bbody & NPEX & FDcut & CPL \\
\hline$N_{\mathrm{H}, 1}\left(10^{22} \mathrm{~cm}^{-2}\right)$ & $6.1 \pm 0.7$ & $4.1_{-2.1}^{+1.1}$ & $4.0 \pm 0.7$ & $3.7_{-1.2}^{+0.9}$ & $4.1_{-0.8}^{+0.9}$ & $3.9 \pm 1.0$ \\
\hline$N_{\mathrm{H}, 2}\left(10^{22} \mathrm{~cm}^{-2}\right)$ & - & $17_{-11}^{+13}$ & - & $27_{-21}^{+20}$ & $21_{-7}^{+6}$ & $19 \pm 8$ \\
\hline$C F$ & - & $0.36_{-0.13}^{+0.42}$ & - & $0.21_{-0.09}^{+0.31}$ & $0.45_{-0.08}^{+0.09}$ & $0.42_{-0.10}^{+0.16}$ \\
\hline $\mathcal{F}\left(10^{-11} \mathrm{erg} \mathrm{cm}^{-2} \mathrm{~s}^{-1}\right)^{a}$ & $8.99 \pm 0.18$ & $9.9 \pm 0.6$ & $7.7 \pm 0.5$ & $9.5_{-0.5}^{+0.6}$ & $10.49_{-0.11}^{+0.44}$ & $10.2_{-0.4}^{+0.5}$ \\
\hline$A_{2}$ & - & - & - & $\left(7.9_{-2.5}^{+2.9}\right) \times 10^{-7}$ & - & - \\
\hline$\Gamma$ & $1.65_{-0.05}^{+0.06}$ & $1.78 \pm 0.09$ & $1.48_{-0.10}^{+0.11}$ & $1.11 \pm 0.06$ & $1.82 \pm 0.06$ & $1.68_{-0.07}^{+0.08}$ \\
\hline$E_{\text {cut }}(\mathrm{keV})$ & $7.1_{-0.5}^{+0.6}$ & $7.6 \pm 0.7$ & $5.5_{-0.7}^{+1.0}$ & - & $<3.9$ & - \\
\hline$E_{\text {fold }}(\mathrm{keV})$ & $27.6_{-2.2}^{+2.6}$ & $33_{-4}^{+5}$ & $25.7_{-2.8}^{+3.5}$ & $9.0_{-0.6}^{+0.8}$ & $23.9_{-1.8}^{+2.2}$ & $27.3_{-2.5}^{+3.0}$ \\
\hline$R_{\text {bbody }}(\mathrm{km})$ & - & - & $0.23_{-0.06}^{+0.07}$ & - & - & - \\
\hline$k T(\mathrm{keV})$ & - & - & $1.88_{-0.19}^{+0.21}$ & - & - & - \\
\hline Eqw FeK $\alpha(\mathrm{eV})$ & $18 \pm 12$ & $12_{-12}^{+13}$ & $18_{-14}^{+12}$ & $12_{-12}^{+13}$ & $6_{-7}^{+10}$ & $10_{-10}^{+12}$ \\
\hline$C C_{\mathrm{B}}$ & $1.003 \pm 0.008$ & $1.003 \pm 0.008$ & $1.003 \pm 0.008$ & $1.003 \pm 0.008$ & $1.003 \pm 0.008$ & $1.003 \pm 0.008$ \\
\hline$C C_{\mathrm{XRT}}$ & $0.62 \pm 0.06$ & $0.59 \pm 0.06$ & $0.59 \pm 0.06$ & $0.59 \pm 0.06$ & $0.58 \pm 0.06$ & $0.59 \pm 0.06$ \\
\hline $\mathcal{L}\left(10^{35} \mathrm{erg} \mathrm{s}^{-1}\right)^{b}$ & $5.43 \pm 0.11$ & $6.0 \pm 0.4$ & $4.64 \pm 0.27$ & $5.76_{-0.27}^{+0.32}$ & $6.32_{-0.07}^{+0.27}$ & $6.14_{-0.24}^{+0.27}$ \\
\hline$\chi^{2} /$ d.o.f. & $717.03 / 660$ & $699.44 / 658$ & $694.78 / 658$ & $699.45 / 658$ & $763.48 / 658$ & $743.66 / 659$ \\
\hline$\chi_{\mathrm{red}}^{2}$ & 1.086 & 1.063 & 1.056 & 1.063 & 1.160 & 1.128 \\
\hline
\end{tabular}

Notes. PC denotes models with partial covering, while $C C_{\mathrm{B}}$ and $C C_{\mathrm{XRT}}$ are the cross-calibration constants for $N u S T A R / \mathrm{FPMB}$ and $S w i f t / \mathrm{XRT}$, respectively, with respect to NUSTAR/FPMA. ${ }^{(a)}$ Unabsorbed powerlaw flux between $3-10 \mathrm{keV}$; ${ }^{(b)}$ luminosity between $3-10 \mathrm{keV}$ for a distance of $7.1 \mathrm{kpc}$.

period of $41.287054 \mathrm{~s}$ (for more information about the timing solution, see Sect. 4.2). The Swift data did not provide enough signal to allow for phase-resolved analysis.

The very high resolution in phase-space is possible thanks to NUSTAR's high throughput even at energies above $10 \mathrm{keV}$. We assumed that neither the detector response nor the background changes on timescales of the pulse period, and therefore used the respective phase-averaged information. We rebinned each spectrum to a $\mathrm{S} / \mathrm{N}$ of 5 within ISIS and on average obtained signal up to $22 \mathrm{keV}$ with about 60 bins per spectrum.

For spectral modeling, we used the partially absorbed highecut (without the blackbody component). We confirmed that using the NPEX model does not change our conclusions. Due to the reduced $\mathrm{S} / \mathrm{N}$, we had to simplify the spectral model and fixed the covering fraction, the absorption columns, the cutoff energy, and the folding energy at the respective values of the phase-averaged fit. This approach left us with only two free parameters: the flux and the photon index $(\Gamma)$. Instead of the photon index, we also tried allowing one of the absorption parameters to vary, but this resulted in large degeneracies and unconstrained parameters, due to the lack of coverage at very low energies. We therefore limited ourselves to capturing all observed spectral changes by a variable photon index. The absolute values of the photon index should consequently be taken with a grain of salt, but its variation is a very good tracer of spectral variability and changes in hardness.

The results of this fit are shown in Fig. 2. Overall, the description of the data is statistically very good, with an average $\chi_{\text {red }}^{2}$ of 0.96 . During most of the pulse, the photon index varies rather smoothly. In particular, no strong change is evident in the main peak of the pulse profile or during the low state around phase 0.7 .

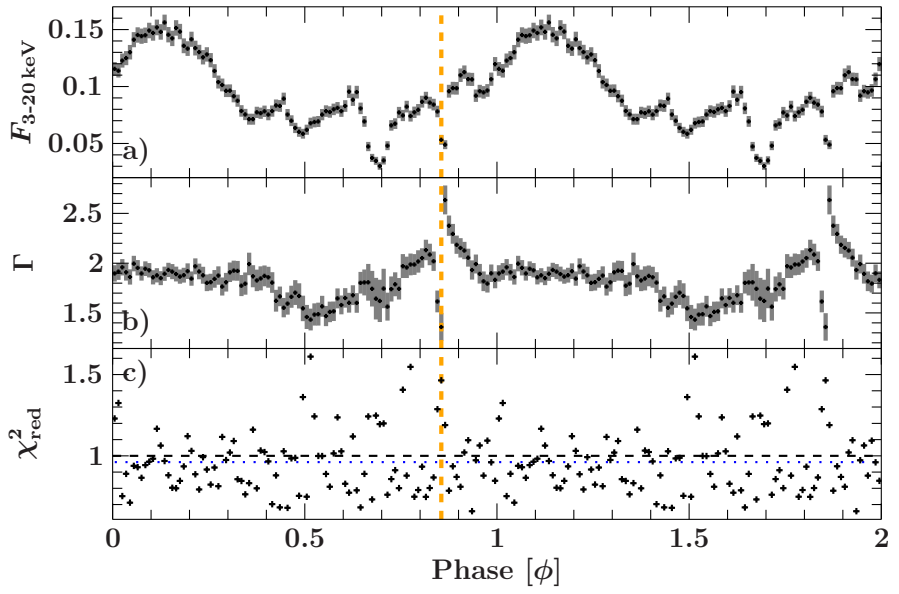

Fig. 2. Results of the phase-resolved spectral fits using 100 phasebins with NUSTAR data only. Panel a: Flux between $3-20 \mathrm{keV}$ in $\mathrm{keV} \mathrm{cm}^{-2} \mathrm{~s}^{-1}$. Panel $b$ : Photon index. All other continuum parameters were frozen to the phase-averaged value. The orange dashed line indicates the dip to guide the eye. Panel $c: \chi_{\text {red }}^{2}$ value for each phase-bin. The blue dotted line indicates the average $\chi_{\text {red }}^{2}$ overall bins.

However, we can clearly make out an interesting feature around phase 0.85 . The spectrum is significantly harder for about two phase-bins and then suddenly jumps from $\Gamma \approx 1.5$ to $\Gamma>2.5$. This behavior is very similar to that observed by Ferrigno et al. (2016), i.e., a hardening followed by a sudden softening. The feature in the NUSTAR data is of a similar duration to that in the XMM-Newton data, between $2-3 \%$ of the pulse period. 

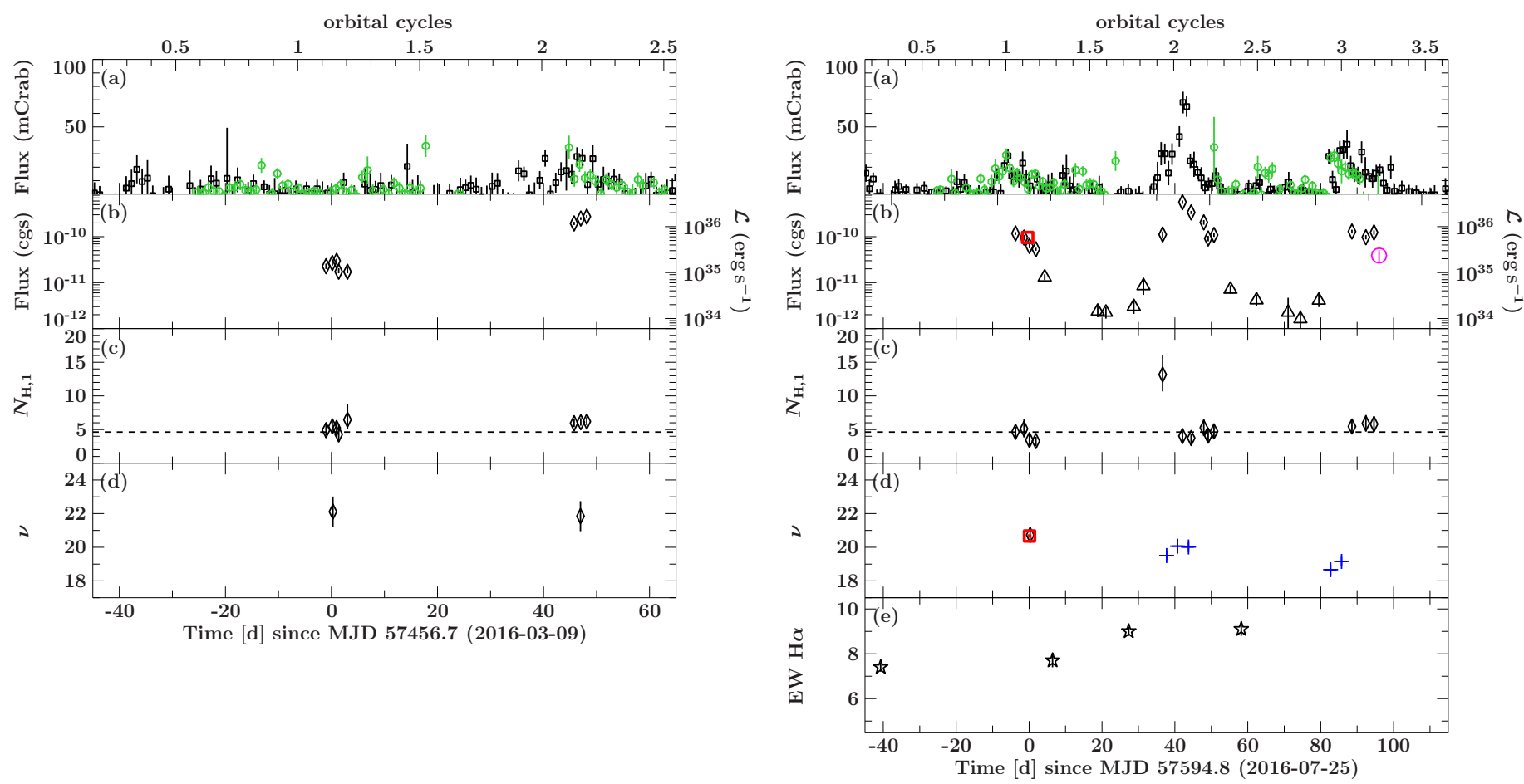

Fig. 3. Panel a: Swift/BAT (black) and MAXI (green) fluxes in mCrab from March to May 2016 (left) and July to October 2016 (right). The fluxes were rescaled to the respective mCrab count-rates, using $0.00022 \mathrm{cts} \mathrm{s}^{-1} \mathrm{~cm}^{-2}(15-50 \mathrm{keV})$ for BAT and $0.0033 \mathrm{cts} \mathrm{s}^{-1} \mathrm{~cm}^{-2}(2-20 \mathrm{keV})$ for MAXI. Panel $b$ : Flux measured in the 3-10 keV energy band in units of $\mathrm{erg} \mathrm{cm}^{-2} \mathrm{~s}^{-1}$. For the data points shown as triangles the absorption was frozen to $5.9 \times 10^{22} \mathrm{~cm}^{-2}$ as it could not be constrained due to the low $\mathrm{S} / \mathrm{N}$. Furthermore, no pulsation search could be performed on those data for the same reason. The NuSTAR flux is shown by a red square and the INTEGRAL/ISGRI flux is shown by a magenta circle and measured between $25-40 \mathrm{keV}$. The $y$-axis is scaled logarithmically. The right hand $y$-scale gives the implied spherical luminosities for a distance of $d=7.1 \mathrm{kpc}$. Panel $c$ : Global absorption column $N_{\mathrm{H}, 1}$ measured with Swift/XRT in $10^{22} \mathrm{~cm}^{-2}$. The columns of NuSTAR and ISGRI were tied to the closest XRT point and are therefore not shown. Panel $d$ : Pulse frequency $v-24.2 \mathrm{mHz}$ in $\mu \mathrm{Hz}$. Blue crosses are values measured by Fermi/GBM, black diamonds are from Swift/XRT, and the red square is the NuSTAR value. Panel e: Equivalent width of the $\mathrm{H} \alpha$ line, as measured in the NOT/ALFOSC spectra.

We also observe indications of a small phase shift between the feature in flux and in the photon index. The value of $\Gamma$ drops one phase-bin before the flux goes down. This phase shift seems to be much smaller in the XMM-Newton data, if present at all (Ferrigno et al. 2016).

\section{Long-term trend}

\subsection{X-ray spectra}

We analyzed Swift/XRT snapshots taken before and after the NUSTAR observation and a serendipitous INTEGRAL observation to put the NuSTAR observation into context. Our first Swift campaign was triggered following the repeated non-detections of Type I outbursts, and covered the periastron passages in March and April 2016. A second campaign between July and September 2016 covered almost two complete orbital cycles (Fig. 3).

As the XRT data do not provide enough information to constrain the continuum parameters at the same time as the absorption column, we fitted them all simultaneously with the NUSTAR and INTEGRAL/ISGRI data (which were taken during an outburst that occurred two orbital cycles after the NuSTAR data), requiring that all spectra have the same photon index, cutoff energy, and folding energy. In this approach, the NUSTAR data provide the main constraints on the continuum. The ISGRI data also probe the high-energy continuum, albeit at a much lower $\mathrm{S} / \mathrm{N}$. They are fully consistent with the NUSTAR data, indicating that the continuum did not change significantly between the two outbursts.
The XRT data, on the other hand, allow us to investigate the behavior of the absorption column, i.e., the parameter to which it is most sensitive. As both NUSTAR and ISGRI lack coverage at energies where the absorption is relevant, we tied their respective absorption columns to the one measured by the respective contemporaneous XRT data. Furthermore, for the very faint XRT spectra that have less than 150 total counts, we set the absorption parameters to the best-fit values shown in Table 1 as they could not be constrained from the data.

In our partial covering model, we have three parameters to describe the complex absorption: $N_{\mathrm{H}, 1}, N_{\mathrm{H}, 2}$, and the covering fraction. We allowed in turn each of these parameters to be independently fit for each Swift spectrum, while the other two were tied across all data sets (i.e., treated as global parameters as defined by Kühnel et al. 2016). We find equally good fits when allowing either the global column, $N_{\mathrm{H}, 1}$, or the covering fraction $f$ to vary. However, allowing only $N_{\mathrm{H}, 2}$ to vary results in a significantly worse fit.

In Fig. 3c, we show the result for a variable $N_{\mathrm{H}, 1}$. As can be seen, the absorption column is relatively constant over different outbursts and fluxes. We only find one significant outlier at MJD 57631.4 where the column increases drastically to $\left(13.6_{-2.5}^{+2.9}\right) \times 10^{22} \mathrm{~cm}^{-2}$. The results for a variable covering fraction are qualitatively similar; there is a strong increase for the same observation to $f=0.91 \pm 0.06$, while on average we observe $\langle f\rangle=0.56 \pm 0.13$. This outlier observation took place around orbital phase 0.93 , but no other XRT observation covers this exact orbital phase. 


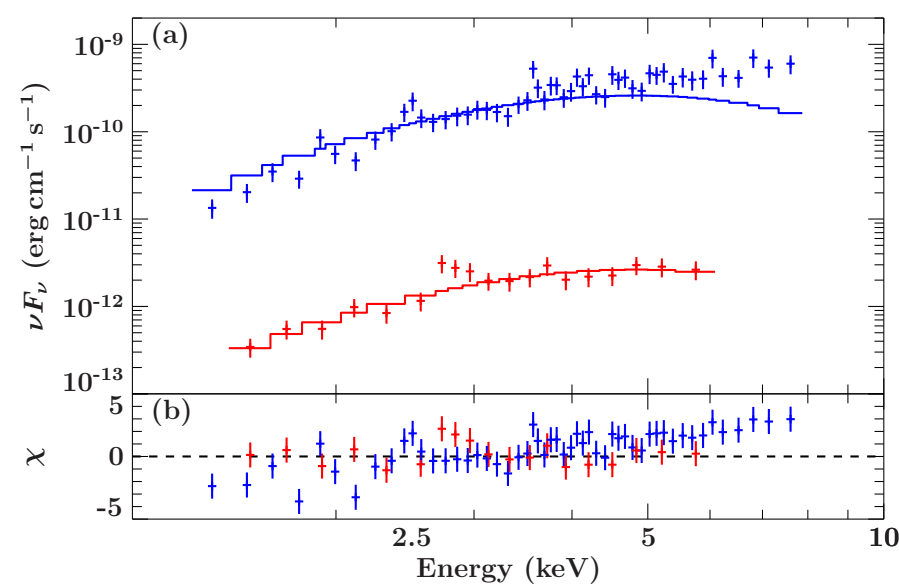

Fig. 4. Swift/XRT spectra and residuals of the combined faint states (red) and during a single bright observation (ObsID 00030799031, blue). The model is the best-fit blackbody spectrum fitted to the faint state (see Table 2), and only scaled in flux to match the brighter state. The brighter state is clearly harder than the faint data.

The XRT data show that even in March 2016, when neither BAT nor MAXI measured residual flux, the source had not turned off completely but was still showing a luminosity around $(1.4 \pm 0.2) \times 10^{35} \mathrm{erg} \mathrm{s}^{-1}$ between $3-10 \mathrm{keV}$. This luminosity was very likely dominated by accretion, as shown by the hard X-ray spectrum and the still remaining pulsations (see Sect. 4.2).

The lowest luminosity during the Swift campaign was observed on MJD 57669 with $L_{\mathrm{X}}=\left(1.0_{-0.5}^{+0.6}\right) \times 10^{34} \mathrm{erg} \mathrm{s}^{-1}$. However, the very low count-rate did not allow us to search for pulsations. The spectral shape is consistent with the other XRT spectra, but could not be individually constrained.

To obtain a better understanding of the spectrum at low fluxes, we therefore combined all spectra with a $3-10 \mathrm{keV}$ flux less than $5 \times 10^{-11} \mathrm{erg} \mathrm{cm}^{-2} \mathrm{~s}^{-1}$ (marked by triangles in Fig. 3) into an average low flux spectrum. We describe the spectrum with a model based on the best-fit NUSTAR model with a partially covered continuum with a high-energy cutoff. As a first approach we froze all parameters except the flux to the best-fit NUSTAR values (see Table 1), but we did not find an acceptable fit $\left(\chi_{\text {red }}^{2}=1.24\right.$ for 18 d.o.f.), which indicates that the spectrum changed significantly.

We then allowed either the photon index, one of the absorption columns, or the covering fraction to be variable. This resulted in an improved fit, with $\Delta \chi^{2} \approx 6$ for each of those parameters (the second column of Table 2 shows the case with a variable photon index). We also tried models where pairs of these parameters were allowed to be variable. With this approach, we found that we obtained a best fit when allowing the photon index and the global absorption column $N_{\mathrm{H}, 1}$ to vary. The best-fit parameters are presented in the third column of Table 2.

We also tried to fit the data with just a thermal blackbody model, and find a slightly better fit (fourth column in Table 2). We find a blackbody temperature of $k T=1.22_{-0.22}^{+0.31} \mathrm{keV}$. This is somewhat hotter than the temperatures Wijnands \& Degenaar (2016) and Tsygankov et al. (2016) found in the quiescent phases of $4 \mathrm{U} 0115+63$ and $\mathrm{V} 0332+53$, but our luminosity is also slightly higher $\left(L_{x}=(2.2 \pm 0.4) \times 10^{34} \mathrm{erg} \mathrm{s}^{-1}\right)$. We find a radial size of the blackbody of $r=280_{-90}^{+140} \mathrm{~m}$, which is at the lower end compared to the values found by Wijnands \& Degenaar (2016).
Table 2. Best-fit model parameters for the faint XRT spectra.

\begin{tabular}{rlll}
\hline \hline Parameter & HighE 1 & HighE 2 & Bbody \\
\hline$N_{\mathrm{H}, 1}\left(10^{22} \mathrm{~cm}^{-2}\right)$ & - & $4.1_{-1.8}^{+2.2}$ & $1.1_{-1.2}^{+1.4}$ \\
$\Gamma / k T(\mathrm{keV})$ & - & $2.2_{-0.7}^{+0.8}$ & $1.22_{-0.22}^{+0.31}$ \\
$R(\mathrm{~m})$ & - & - & $280_{-90}^{+140}$ \\
$\mathcal{L}\left(10^{34} \mathrm{erg} \mathrm{s}^{-1}\right)^{a}$ & $2.83 \pm 0.27$ & $2.4_{-0.4}^{+0.5}$ & $1.55_{-0.30}^{+0.38}$ \\
\hline$\chi^{2} /$ d.o.f. & $22.2 / 18$ & $16.4 / 16$ & $15.2 / 16$ \\
$\chi_{\text {red }}^{2}$ & 1.24 & 1.03 & 0.95 \\
\hline
\end{tabular}

Notes. ${ }^{(a)}$ Luminosity between $3-10 \mathrm{keV}$ for a distance of $7.1 \mathrm{kpc}$.

\subsection{Pulse period}

The pulse period of EXO 2030+375 is normally well monitored by Fermi $/ \mathrm{GBM}^{2}$ (Finger et al. 2009). During the periastron passages between 2016 March and August (MJD 57450 to MJD 57600 ), however, the source was too faint to be measurable by GBM and therefore the behavior of the spin-down was not captured. We therefore searched for periodicity in the NuSTAR and Swift/XRT data obtained during the periastron passages in that time frame. We transferred all time information to the solar barycenter, and corrected for the orbital Doppler shift using the ephemeris of Wilson et al. (2008, Fit 2). We searched for a period around the known value of $41.3 \mathrm{~s}$ in the NUSTAR source event lists, using the epochfolding technique (e.g., Leahy et al. 1983). Uncertainties were estimated through a Monte Carlo simulation (Davies 1990). We find a period of $P_{\text {NuSTAR }}=41.28705 \pm 0.00008 \mathrm{~s}$ ( $1 \sigma$ uncertainty $)$ at epoch $t_{0}=$ MJD 57594.37019 and no evidence for a change in the period $\dot{P}$ over the observation.

To cross-check this value, we used the epoch folding technique on the Swift/XRT light curves taken between MJD 57591 and MJD 57599 . However, in order to avoid strong secondary maxima within the epoch folding result, we split the light curves into segments where gaps in the data resulting from bad time intervals are present. The resulting pulse periods for all segments and observations are consistent with $41.22 \pm 0.13 \mathrm{~s}$.

In order to further increase the precision of the XRT pulse period measurement, we phase connected the pulse profiles of all light curve segments using 16 phase bins (Manchester \& Taylor 1977; Deeter et al. 1981). The resulting phase shift, $\delta \phi$, between each adjacent light curve segment is degenerate by an integer multiple corresponding to the error propagated uncertainty of the pulse period determined by epoch folding. This uncertainty results in a number of possible pulse phase evolutions over the time of the Swift observations.

We consequently fitted each evolution assuming a constant spin-change, i.e., $\delta \phi\left(t-t_{0}\right)=v t+0.5 \dot{v} t^{2}$ with the time of each observation $t$, a reference time $t_{0}=$ MJD 57594.85875 , the pulse frequency $v=1 / P$, and its derivative $\dot{v}=-\dot{P} / P^{2}$, where $\dot{P}$ is the pulse period derivative. After having fitted all possible pulse phase evolutions resulting from the mentioned degeneracy, we selected the evolution providing the best goodness of fit $\left(\Delta \chi^{2}=3.5\right.$ with 6 degree of freedom), which results in a final pulse period of $41.2869 \pm 0.0004 \mathrm{~s}$, in very good agreement

2 https://gammaray.msfc.nasa.gov/gbm/science/pulsars. html 


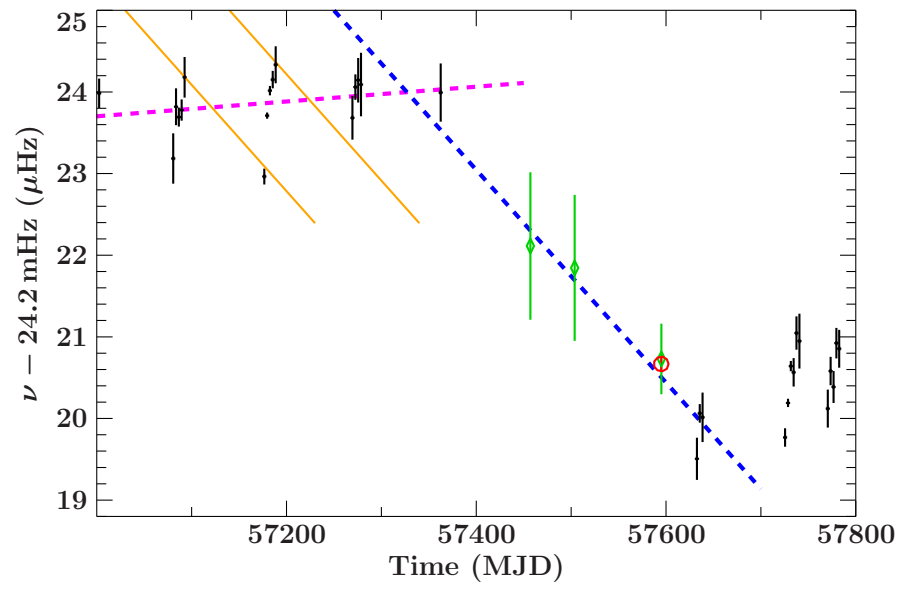

Fig. 5. Pulse frequency evolution of EXO 2030+375, as measured with Fermi/GBM. The NuSTAR measurement is shown as a red circle, the Swift measurements are shown as green diamonds. Each group of black data points corresponds to one Type I outburst visible to GBM. During each outburst, the source spins up, while between outbursts it spins down again. With the start of the quiescence period, a continuous spin-down started. The dashed lines are linear regression fits to the active phase, which shows a secular spin-up trend (magenta), and to the spin-down in quiescence (blue), respectively. The orange solid lines have the same slope as the best-fit spin-down linear regression to guide the eye between the spin-up episodes during outbursts. The data after MJD 57700.0 show that with the again stronger outbursts, EXO 2030+375 is also starting to spin-up again.

with NUSTAR. Our best-fit period derivative, $\dot{P}$, is consistent with zero, which is due to the short time range covered here.

We used a similar approach for the previous two periastron passages where we also had Swift data available (around MJD 57451 and 57 493). As the source was overall much fainter, the uncertainties on the obtained periods were higher. We find $41.2846 \pm 0.0008 \mathrm{~s}$ on MJD 57457.0 and $41.2850 \pm 0.0008 \mathrm{~s}$ on MJD 57 503.7, again with no evidence for $\dot{P}$ within each epoch.

Folding the Swift/XRT data taken during apastron did not result in a significant detection of pulsations at the $99 \%$ level when taking the number of trials into account (based on the $L$-statistic, Davies 1990). Given the low photon counts in these data, the non-detections are not surprising and do not necessarily imply that the source is not pulsing. From a rough estimate of the detectable pulsed fraction (Brazier 1994), we find that the data are only sensitive to pulsed fractions $>75 \%$, while the pulsed fraction during the periastron observations is only $\sim 40 \%$ in XRT.

The NUSTAR and Swift results are put into context with the Fermi/GBM results in Fig. 5. As can be seen, our results confirm an almost linear long-term spin-down with a rate of change of $\dot{P}=(2.7814 \pm 0.0003) \times 10^{-10} \mathrm{~s} \mathrm{~s}^{-1}$. This spin-down is of a comparable strength to the one typically observed between outbursts (see Fig. 5). This indicates that the accretion rate was very low during the whole quiescent epoch and likely not higher than during typical apastron passages.

\subsection{Optical monitoring}

Between June and September 2016, we obtained four measurements of the $\mathrm{H} \alpha$ line with the NOT to trace the evolution of the circumstellar disk. We show the shape of the $\mathrm{H} \alpha$ line in Fig. 6. We measure equivalent widths (EWs) between 7-9 $\AA$ in all four spectra and show their evolution in Fig. 3e. Similar values were measured by Reig et al. (1998) and Wilson et al. (2002), just

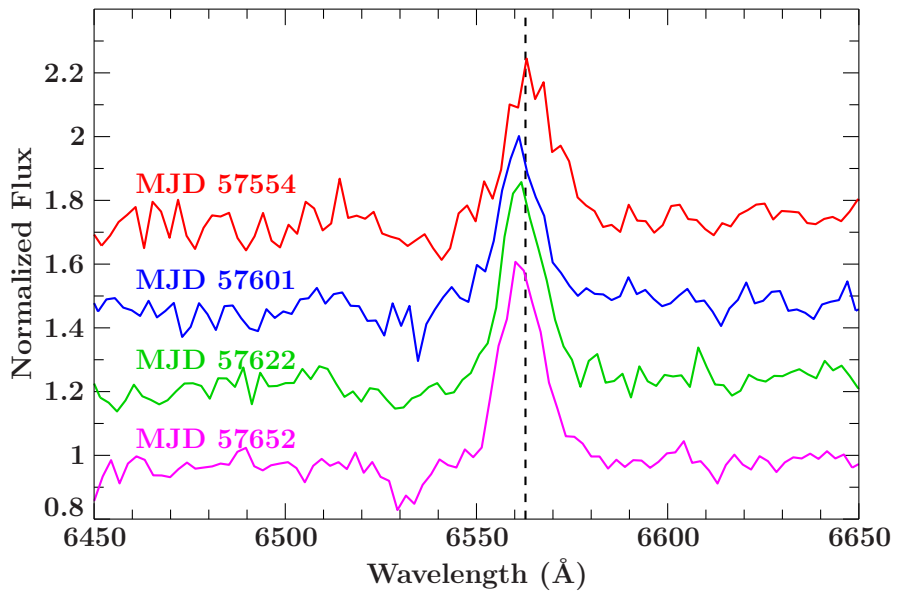

Fig. 6. $\mathrm{H} \alpha$ line of EXO $2030+375$ as measured with the NOT/ALFOSC on four different nights between June and September 2016. The spectra are shifted in steps of 0.25 in flux for visual clarity. The dashed line indicates the rest wavelength of $\mathrm{H} \alpha$.

after the period when there were also missing X-ray outbursts (MJD $\lesssim 50000$ ), and coinciding with a change in orbital phase of the peak of the outbursts (Laplace et al. 2017). These events, and the ones we present here, are separated by about $21 \mathrm{yr}$, roughly the same separation as for the Type II outbursts.

These similar periodicities could be explained by KozaiLidov oscillations with a 21-yr period (Laplace et al. 2017). The Kozai-Lidov effect (Kozai 1962; Lidov 1962) causes an initially misaligned accretion disk to oscillate between a larger eccentricity and a larger inclination with respect to the orbital plane. As the observed EW is directly connected to the inclination of the circumstellar disk with the line of sight, the periodic occurrence of low EWs, coincident with a low X-ray state, could be explained by a change in inclination on the Kozai-Lidov timescale. The EW therefore does not necessarily trace the size or density of the disk, but only its projected area. As Laplace et al. (2017) argue, it is likely that the faint-states and the change in outburst phase are connected with a highly inclined disk (with respect to the orbital plane), which is observed as a low $\mathrm{EW}$ of the $\mathrm{H} \alpha$ line. On the other hand, the giant outbursts coincide with a high eccentricity of the disk and a low inclination, resulting in a much larger EW.

We note, however, that Baykal et al. (2008) also measured low values of the EWs after the giant Type II outburst in 2006 June. As the Kozai-Lidov oscillation would predict a lower inclination and therefore higher $\mathrm{EW}$ of the $\mathrm{H} \alpha$ line during that time, this measurement might be instead explained by a disk-loss produced by the large quantity of accreted material.

The line profiles we observe show marginal indications for a P Cygni profile, with an estimated velocity of $\sim 1300 \mathrm{~km} \mathrm{~s}^{-1}$. If this profile were real, it would indicate the presence of a strong stellar wind, which would be responsible for the observed absorption. However, the low $\mathrm{S} / \mathrm{N}$ of the data prevents us from drawing any further conclusions.

\section{Discussion}

\subsection{Spectral shape}

We find that a partially covered highecut model describes the phase-averaged NuSTAR and contemporaneous XRT spectrum very well (Table 1). A similar model has traditionally been used to describe the spectrum of EXO 2030+375, e.g., by 
Reynolds et al. (1993) and Wilson et al. (2008). All these works have found a consistent correlation between luminosity and photon index, i.e., softer spectra at higher luminosities. Reig \& Coe (1999) find that this correlation levels off at lower luminosities around $10^{36} \mathrm{erg} \mathrm{s}^{-1}$. In our data, however, we find a much softer photon index than Reig \& Coe $\left(1999 ; \Gamma=1.81_{-0.10}^{+0.08}\right)$, thereby reversing the typical correlation. This is somewhat reminiscent of the behavior found in black hole binaries, which in the hard state show the hardest photo index, typically around $1 \%$ of the Eddington luminosity, and soften significantly towards lower and higher fluxes (e.g., Tomsick et al. 2001; Yang et al. 2015). Depending on the bolometric corrections and mass of the neutron star, the lowest luminosities observed by Reig \& Coe (1999) of around $2 \times 10^{36} \mathrm{erg} \mathrm{s}^{-1}$ correspond to about $1 \%$ Eddington.

On the other hand, Wilson et al. (2008) observed a relatively stable cutoff energy around $12.5 \mathrm{keV}$ until the end of the outburst, where it dropped to values around $10 \mathrm{keV}$ at the lowest fluxes. In our data we find an even lower cutoff energy of $6.8 \pm 0.5 \mathrm{keV}$ (Table 1), which seems to agree roughly with the trend found by Wilson et al. (2008) towards the end of the outburst and therefore does not require a different behavior at very low luminosities.

Klochkov et al. (2007) also use a highecut to describe INTEGRAL data taken during the 2006 giant outburst. They find an even higher cutoff energy around $20-25 \mathrm{keV}$, depending on the exact model. The main difference between the INTEGRAL spectral shape and the one we find at low luminosities with $N U S T A R$ is the curvature between $\sim 5-30 \mathrm{keV}$, which is much less pronounced in our data. This could be related to the possible presence of a cyclotron resonant scattering feature (CRSF) or a $10 \mathrm{keV}$ bump in the brighter INTEGRAL data (Klochkov et al. 2007). Wilson et al. (2008), on the other hand, find evidence for an absorption feature around $10 \mathrm{keV}$, which they interpret as a CRSF.

We tried to include a CRSF or $10 \mathrm{keV}$ bump, but do not find any evidence for such a feature in that energy range. If we assume a CRSF with the energy and width fixed to the values found by Wilson et al. (2008, $E=11.44 \mathrm{keV}$ and $\sigma=3.08 \mathrm{keV})$ we can put a stringent upper limit on the optical depth ${ }^{3} \tau \leq 0.012$, which is about one order of magnitude below the best-fit value found by Wilson et al. (2008).

Klochkov et al. (2008) measured a CRSF with INTEGRAL at $64 \mathrm{keV}$ during a giant outburst. While the source is detected in the phase-averaged NUSTAR data up to $\sim 70 \mathrm{keV}$, we do not find evidence for this CRSF. If we assume an energy of $64 \mathrm{keV}$ and a width of $5 \mathrm{keV}$, we can put an upper limit on the optical depth $\tau<0.07$. This is significantly smaller than the typically observed depths (e.g., the harmonic line in Vela X-1 at $55 \mathrm{keV}$ with $\tau \approx 0.3$, Fürst et al. 2014). However, as we do not have coverage above the proposed CRSF energy, it is still possible that the line is subsumed in our continuum model. As the Swift monitoring data suggest a high magnetic field, it is also possible that the CRSF energy moved out of the observable NUSTAR range with the lower luminosity (for a possible luminosity dependence of the CRSF energy see, e.g., Becker et al. 2012).

\subsection{Propeller effect and thermal emission}

Previous studies (Reynolds et al. 1996; Wilson et al. 2002) have postulated that the propeller effect might occur at luminosities below $10^{36} \mathrm{erg} \mathrm{s}^{-1}$. This effect would shut off most accretion and

\footnotetext{
$\tau$ is total optical depth of the line and not the line strength $d$, as de-
} fined in the gabs model. They are connected via $\tau=d /(\sigma \sqrt{2 \pi})$. result in a strong drop in the luminosity. However, in the NuSTAR and XRT data we observe pulsations and a hard spectrum at luminosities as low as $1.4 \times 10^{35} \mathrm{erg} \mathrm{s}^{-1}$ indicating that accretion is still ongoing and channeled by the $B$-field. Our results therefore reduce the upper limit for the propeller effect by almost an order of magnitude.

Analyzing low-luminosity data of A $0535+26$, Rothschild et al. (2013) find that in this source pulsations are sometimes present at luminosities as low as $2 \times 10^{34} \mathrm{erg} \mathrm{s}^{-1}$. As the postulated cyclotron line energies and therefore magnetic fields in the two sources are similar, we might expect to observe pulsations in EXO 2030+375 at even lower luminosities. These low luminosities are sustainable by just the wind-loss rate of the Be companion, even if no accretion from the circumstellar disk occurs.

The lowest luminosities we observed were $\left(1.0_{-0.5}^{+0.6}\right) \times$ $10^{34} \mathrm{erg} \mathrm{s}^{-1}$. However, at these low fluxes, the data are not statistically sensitive to pulsations at the typical observed level, and we therefore cannot determine if they are still present. However, by averaging over all low flux observations we find that the spectrum is very soft and well described by a purely blackbody continuum with a temperature of $k T=1.22_{-0.22}^{+0.31} \mathrm{keV}$. This softening could indicate that the source has actually entered the propeller regime and we are only observing the neutron star surface (or only the hot spots at the magnetic poles) that are cooling. This idea has also been discussed by Wijnands \& Degenaar (2016) and Tsygankov et al. (2016) for low-luminosity observations of V $0332+53$ and 4U $0115+63$ and by Reig et al. (2014) for SAX J2103.5+454.

We note that the unabsorbed continuum flux before each periastron passage is slowly rising, while still being relatively low. A rising flux, however, cannot be explained by neutron star cooling, and we therefore infer that some accretion power needs to contribute to the observed luminosity. Furthermore, the decline curve does not seem to follow the expected cooling curve of the neutron star surface because it is also likely influenced by residual accretion. Nonetheless, we find strong indications that the spectral shape is significantly softer and that the luminosity seems to drop to the low levels rather abruptly.

If we assume that accretion has stopped during the low flux state, the onset of the propeller effect happens somewhere between $2 \times 10^{34} \mathrm{erg} \mathrm{s}^{-1}$ and $1 \times 10^{35} \mathrm{erg} \mathrm{s}^{-1}$. The propeller effect takes place when the magnetospheric radius is larger than the corotation radius (Illarionov \& Sunyaev 1975; Cui 1997; Campana et al. 2002), resulting in a critical luminosity $L_{\text {prop }}$ of

$L_{\text {prop }}=7.3 \times k^{7 / 2} P^{-7 / 3} R_{6}^{5} B_{12}^{2} M_{1.4}^{-2 / 3} \times 10^{37} \mathrm{erg} \mathrm{s}^{-1}$,

where $B_{12}$ is the magnetic field strength in $10^{12} \mathrm{G}, P$ is the pulse period in seconds, $M_{1.4}$ is the neutron star mass in $1.4 M_{\odot}$, and $R_{6}$ is the neutron star radius in $10^{6} \mathrm{~cm}$. The factor $k$ parameterizes the effective coupling radius of the accretion disk to the magnetic field, which we set to 1 here (Wang 1996). For a derivation of this expression see Appendix A.

The critical luminosity obviously depends strongly on the magnetic field strength. Assuming a magnetic field strength of around $5 \times 10^{12} \mathrm{G}$ at the pole, as inferred from the $64 \mathrm{keV}$ CRSF measured by Klochkov et al. (2007), translates to a field of about $2.5 \times 10^{12} \mathrm{G}$ at the equator, where the accretion disk interacts with the magnetic field. This field strength would put the onset of the propeller effect around $8.8 \times 10^{34} \mathrm{erg} \mathrm{s}^{-1}$, exactly in the expected range. However, if we assume $k=0.5$, which is often done for simple accretion disks (e.g., Ghosh \& Lamb 1979), we 
find $L_{\text {prop }} \approx 7 \times 10^{33} \mathrm{erg} \mathrm{s}^{-1}$, which is below the lowest flux data of Swift.

If we assume the much lower field of $1 \times 10^{12} \mathrm{G}$ postulated by Wilson et al. (2008), the luminosity for the onset of the propeller effect would drop to below $2 \times 10^{33} \mathrm{erg} \mathrm{s}^{-1}$ in the spherical case and to $\approx 2 \times 10^{32}$ for $k=0.5$. Such a low field seems therefore unlikely to be consistent with our observations.

However, we note that the calculation of the propeller luminosity is strongly dependent on the accretion flow, and therefore contains a rather large systematic uncertainty (see also Appendix A). In particular the calculation is based on spherical accretion, which is not necessarily a good approximation for EXO 2030+375. Any value should therefore be taken with caution, but the rough estimate results in values consistent with the inferred luminosity for the onset of the propeller effect from the Swift/XRT data.

Only in the higher magnetic field case can EXO 2030+375 enter the propeller regime at all, according to the model presented by Tsygankov et al. (2017). In this model a cool, nearly unionized accretion disk can form for sufficiently slow rotators and weak magnetic field strengths. This disk will penetrate deeper into the magnetosphere and can sustain persistent accretion at a low level. Following Eq. (7) of Tsygankov et al. (2017) we find that the neutron star would have to spin with a period of $20 \mathrm{~s}$ or faster to enter the propeller regime assuming a field of $\sim 1 \times 10^{12} \mathrm{G}$, but this is well below the critical period for all realistic values of $k$ in the high magnetic field case. This again shows that our observations are more consistent with a high magnetic field.

\subsection{Accretion geometry}

The phase-resolved analysis revealed a feature similar to that observed in XMM-Newton, during which the photon index rapidly changes for about $3 \%$ of the pulse period. Ferrigno et al. (2016) interpreted this feature as part of the accretion column passing through the line of sight, resulting in a significant rise in absorption column and in a harder spectrum, due to more reprocessing of the emerging radiation. With $N u S T A R$, we are not able to probe variations in $N_{\mathrm{H}}$, due to the limited coverage at low energies. Ferrigno et al. (2016) further argue that the hotspot size should scale with luminosity as $\phi \propto L^{1 / 7}$, which would result in a smaller hotspot in the NUSTAR observations. However, we do not observe this effect; instead, the duration of the feature in NUSTAR seems to be similar to or longer than that in XMMNewton. This indicates either that the accretion column size is not following the expectations or that the feature is independent of the accretion column.

A duration of $2 \%$ implies a size of around $1.3 \mathrm{~km}$ at the equator of the neutron star (assuming a canonical radius of $10 \mathrm{~km}$ ). However, as the accretion column is likely at higher latitudes than the equator, this number can only be regarded as an upper limit. For a detailed interpretation, other effects (e.g., lightbending due to the strong gravity in the vicinity of the neutron star) have to be taken into account, which are beyond the scope of this paper.

During the brighter observations of the Swift/XRT monitoring we find a very stable spectrum, with an average absorption column of $N_{\mathrm{H}, 1}=6.1 \times 10^{22} \mathrm{~cm}^{-2}$. Only one XRT observation shows a significantly enhanced $N_{\mathrm{H}}$ value. This is similar to the behavior seen in GX 304-1, which also showed enhanced absorption close to the periastron passage in some orbits (Rothschild et al. 2017). This was interpreted as a warped and precessing Be-disk passing through the line of sight
(Kühnel et al. 2017). However, the available EXO 2030+375 data do not allow us to put constraints on the precession frequency or size of the Be-disk; these parameters are likely different from the long-term oscillation due to the Kozai-Lidov effect discussed by Laplace et al. (2017), as those timescales are much longer. From optical monitoring we can rule out a drastically increased Be-disk, as the equivalent width of the $\mathrm{H} \alpha$ line is constantly low.

\section{Conclusion}

We have presented an analysis of NuSTAR, Swift, and INTEGRAL data, supported by optical spectra with the NOT, and taken at very low luminosities of EXO 2030+375. These data provide an in-depth look into the low-luminosity regime of this accreting neutron star. The NUSTAR data were taken at a luminosity around $(1.4 \pm 0.2) \times 10^{35} \mathrm{erg} \mathrm{s}^{-1}$ in March 2016 (MJD 57 457). In these data we still observe a hard spectrum and pulsations, clear evidence of ongoing accretion. We confirm the sudden change in spectral hardness found over a narrow range of pulse phases in the XMM-Newton data in phase-resolved spectroscopy (Ferrigno et al. 2016), indicating that the physical reason for this feature is independent of luminosity. More detailed investigations at more luminosity levels are necessary to understand its origin; correct modeling of the relativistic effects around the neutron star is also necessary (see, e.g., Kraus et al. 2003; Falkner et al. 2016).

The lowest overall observed luminosity was around $2 \times$ $10^{34} \mathrm{erg} \mathrm{s}^{-1}$ (August 2016) with Swift/XRT. In this observation the spectrum was much softer. We interpret this as the onset of the propeller effect at luminosities just below $10^{35} \mathrm{erg} \mathrm{s}^{-1}$, which is in agreement with a strong magnetic field of $5 \times 10^{12} \mathrm{G}$ or more, as proposed by Klochkov et al. (2008). We cannot constrain the spectral shape well at the lowest luminosities, but find that it is well described by a thermal spectrum with a temperature of around $1.2 \mathrm{keV}$. We interpret this spectrum as possibly originating from the cooling surface of the neutron star. As EXO 2030+375 has now picked up its regular outburst behavior again, further observations at these low luminosities are difficult. Nonetheless, with the expected 21-yr periodic behavior (Laplace et al. 2017), another low-activity state might occur during the lifetime of Athena, which will also provide the necessary sensitivity to study the apastron spectrum in detail.

Acknowledgements. We thank the referee for the useful comments that helped to improve the paper. We would like to thank the NuSTAR PI, Fiona Harrison, for accepting our observations in Director's Discretionary Time, and the schedulers and the SOCs of Swift and NUSTAR for making them possible. We are grateful for the support Neil Gehrels showed us during this and many other projects. May he rest in peace. F.F. is supported by a European Space Agency (ESA) Research Fellowship at the European Space Astronomy Centre (ESAC), in Madrid, Spain. J.J.E.K. acknowledges support from the Academy of Finland grants 268740 and 295114 and the ESA research fellowship program. This work was supported under NASA Contract No. NNG08FD60C, and made use of data from the NuSTAR mission, a project led by the California Institute of Technology, managed by the Jet Propulsion Laboratory, and funded by the National Aeronautics and Space Administration. This research has made use of the NuSTAR Data Analysis Software (NuSTARDAS) jointly developed by the ASI Science Data Center (ASDC, Italy) and the California Institute of Technology (USA). This work made use of data supplied by the UK Swift Science Data Centre at the University of Leicester. We would like to thank John E. Davis for the slxfig module, which was used to produce all the figures in this work. This research has made use of MAXI data provided by RIKEN, JAXA, and the MAXI team. The Swift/BAT transient monitor results were provided by the Swift/BAT team. The data presented here were obtained in part with ALFOSC, which is provided by the Instituto de Astrofísica de Andalucia (IAA) under a joint agreement with the University of Copenhagen and NOTSA. IRAF is distributed by the National Optical Astronomy 
Observatories, which are operated by the Association of Universities for Research in Astronomy, Inc., under cooperative agreement with the National Science Foundation.

\section{References}

Baykal, A., Kızıloğlu, Ü., Kızıloğlu, N., et al. 2008, A\&A, 479, 301 Becker, P. A., Klochkov, D., Schönherr, G., et al. 2012, A\&A, 544, A123 Brazier, K. T. S. 1994, MNRAS, 268, 709

Burrows, D. N., Hill, J. E., Nousek, J. A., et al. 2005, SSRv, 120, 165

Campana, S., Stella, L., Israel, G. L., et al. 2002, ApJ, 580, 389

Coe, M. J., Payne, B. J., Longmore, A., \& Hanson, C. G. 1988, MNRAS, 232, 865

Courvoisier, T. J. L., Walter, R., Beckmann, V., et al. 2003, A\&A, 411, L53

Cui, W. 1997, ApJ, 482, L163

Davies, S. R. 1990, MNRAS, 244, 93

Deeter, J. E., Boynton, P. E., \& Pravdo, S. H. 1981, ApJ, 247, 1003

Elsner, R. F., \& Lamb, F. K. 1977, ApJ, 215, 897

Falkner, S., Schwarm, F. W., Wolff, M. T., et al. 2016, in AAS/High Energy Astrophysics Division, 15, 201.08

Ferrigno, C., Pjanka, P., Bozzo, E., et al. 2016, A\&A, 593, A105

Finger, M. H., Beklen, E., Narayana Bhat, P., et al. 2009, in Proc. of the 2009 Fermi Symposium, eConf Proceedings C091122

Fürst, F., Pottschmidt, K., Wilms, J., et al. 2014, ApJ, 780, 133

Fürst, F., Grinberg, V., Tomsick, J. A., et al. 2016a, ApJ, 828, 34

Fürst, F., Wilson-Hodge, C. A., Kretschmar, P., et al. 2016b, ATel, 8835

Gehrels, N., Chincarini, G., Giommi, P., et al. 2004, ApJ, 611, 1005

Ghosh, P., \& Lamb, F. K. 1979, ApJ, 232, 259

Harrison, F. A., Craig, W., Christensen, F., et al. 2013, ApJ, 770, 103

Houck, J. C., \& Denicola, L. A. 2000, in Astronomical Data Analysis Software and Systems IX, eds. N. Manset, C. Veillet, \& D. Crabtree, ASP Conf. Ser., 216, 591

Illarionov, A. F., \& Sunyaev, R. A. 1975, A\&A, 39, 185

Klochkov, D., Horns, D., Santangelo, A., et al. 2007, A\&A, 464, L45

Klochkov, D., Santangelo, A., Staubert, R., \& Ferrigno, C. 2008, A\&A, 491, 833

Kozai, Y. 1962, AJ, 67, 591

Kraus, U., Zahn, C., Weth, C., \& Ruder, H. 2003, ApJ, 590, 424

Kretschmar, P., Fürst, F., Wilson-Hodge, C. A., et al. 2016, ATel, 9485

Kretschmar, P., Fürst, F., Wilson-Hodge, C. A., et al. 2017, in PoS, Proc. of the INTEGRAL workshop

Kühnel, M., Falkner, S., Grossberger, C., et al. 2016, Acta Polytechnica, 56, 41
Kühnel, M., Rothschild, R. E., Okazaki, A. T., et al. 2017, MNRAS, 471, 1553

Lamb, F. K., Pethick, C. J., \& Pines, D. 1973, ApJ, 184, 271

Laplace, E., Mihara, T., Takagi, T., et al. 2016, ATel, 9263

Laplace, E., Mihara, T., Moritani, Y., et al. 2017, A\&A, 597, A124

Leahy, D. A., Elsner, R. F., \& Weisskopf, M. C. 1983, ApJ, 272, 256

Lebrun, F., Leray, J. P., Lavocat, P., et al. 2003, A\&A, 411, L141

Lidov, M. L. 1962, Planet. Space Sci., 9, 719

Manchester, R. N., \& Taylor, J. H. 1977, Pulsars (San Francisco, USA: W. H. Freeman \& Co. Ltd)

Martin, R. G., Nixon, C., Lubow, S. H., et al. 2014, ApJ, 792, L33

Mihara, T., Makishima, K., \& Nagase, F. 1998, Adv. Space Res., 22, 987

Müller, S., Ferrigno, C., Kühnel, M., et al. 2013, A\&A, 551, A6

Naik, S., Maitra, C., Jaisawal, G. K., \& Paul, B. 2013, ApJ, 764, 158

Negueruela, I., Reig, P., Coe, M. J., \& Fabregat, J. 1998, A\&A, 336, 251

Okazaki, A. T., \& Negueruela, I. 2001, A\&A, 377, 161

Parmar, A. N., White, N. E., \& Stella, L. 1989a, ApJ, 338, 373

Parmar, A. N., White, N. E., Stella, L., et al. 1989b, ApJ, 338, 359

Reig, P., \& Coe, M. J. 1999, MNRAS, 302, 700

Reig, P., Stevens, J. B., Coe, M. J., \& Fabregat, J. 1998, MNRAS, 301, 42

Reig, P., Doroshenko, V., \& Zezas A. 2014, MNRAS, 445, 1314

Reynolds, A. P., Parmar, A. N., \& White, N. E. 1993, ApJ, 414, 302

Reynolds, A. P., Parmar, A. N., Stollberg, M. T., et al. 1996, A\&A, 312, 872

Rothschild, R., Markowitz, A., Hemphill, P., et al. 2013, ApJ, 770, 19

Rothschild, R. E., Kühnel, M., Pottschmidt, K., et al. 2017, MNRAS, 466, 2752

Tanaka, Y. 1986, Radiation Hydrodynamics in Stars and Compact Objects, eds. D. Mihalas, \& K. H. A. Winkler, Proc. IAU Colloq., 89, 255

Tody D., 1993, Astronomical Data Analysis Software and Systems II, eds. R. J. Hanisch, R. J. V. Brissenden, J. Barnes, ASP Conf. Ser., 52, 173

Tomsick, J. A., Corbel, S., \& Kaaret, P. 2001, ApJ, 563, 229

Tsygankov, S. S., Lutovinov, A. A., Doroshenko, V., et al. 2016, A\&A, 593, A16

Tsygankov, S. S., Mushtukov, A. A., Suleimanov, V. F., et al. 2017, A\&A, in press, DOI: 10.1051/0004-6361/201630248

Verner, D. A., Ferland, G. J., Korista, K. T., \& Yakovlev, D. G. 1996, ApJ, 465, 487

Walton, D. J., Tomsick, J. A., Madsen, K. K., et al. 2016, ApJ, 826, 87

Wang, Y. M. 1996, ApJ, 465, L111

Wijnands, R., \& Degenaar, N. 2016, MNRAS, 463, L46

Wilms, J., Allen, A., \& McCray, R. 2000, ApJ, 542, 914

Wilson, C. A., Finger, M. H., Coe, M. J., et al. 2002, ApJ, 570, 287

Wilson, C. A., Fabregat, J., \& Coburn, W. 2005, ApJ, 620, L99

Wilson, C. A., Finger, M. H., \& Camero-Arranz, A. 2008, ApJ, 678, 1263

Winkler, C., Courvoisier, T. J. L., Di Cocco, G., et al. 2003, A\&A, 411, L1

Yang, Q. X., Xie, F. G., Yuan, F., et al. 2015, MNRAS, 447, 1692 


\section{Appendix A: Calculation of the propeller luminosity}

We based our calculations on the seminal works by Lamb et al. (1973), Elsner \& Lamb (1977), and Ghosh \& Lamb (1979), and the corresponding assumptions therein. In particular, we assume spherical accretion with the velocity $v(r)$ at the magnetospheric radius being equal to the freefall velocity $v_{\mathrm{ff}}(r)$ :

$v(r)=v_{\mathrm{ff}}(r)=\sqrt{2 G M / r}$.

Here, $M$ is the mass of the neutron star and $G$ is the gravitational constant. The magnetospheric radius is defined as the radius $r_{\mathrm{m}}$ at which the pressure of the magnetic field $B$ becomes similar to the pressure of the infalling matter with density $\rho$ :

$\frac{B\left(r_{\mathrm{m}}\right)^{2}}{8 \pi}=\rho\left(r_{\mathrm{m}}\right) v\left(r_{\mathrm{m}}\right)^{2}$.

It should be noted that we assume steady accretion, as is commonly done in the literature, and not accretion driven through Rayleigh-Taylor instabilities (see discussion in Elsner \& Lamb 1977). The latter case, with magnetic pressure expressed as $B\left(r_{\mathrm{m}}\right)^{2} / 4 \pi$, would result in a factor of two larger luminosities, which is well within the systematic uncertainties of this estimate.

Using the continuity equation ${ }^{4} \dot{M}=4 \pi \rho r^{2} v(r)$ and the expression of the magnetic field strength at the magnetospheric radius $B=\mu / r_{\mathrm{m}}^{3}$, we can write

$\frac{\mu^{2}}{8 \pi r_{\mathrm{m}}^{6}}=\frac{\dot{M}}{4 \pi r_{\mathrm{m}}^{2} v\left(r_{\mathrm{m}}\right)} v\left(r_{\mathrm{m}}\right)^{2}=\frac{\dot{M}}{4 \pi r_{\mathrm{m}}^{2}} \sqrt{\frac{2 G M}{r_{\mathrm{m}}}}$.

Solving for $r_{\mathrm{m}}$ we find

$r_{\mathrm{m}}=\dot{M}^{-2 / 7} \mu^{4 / 7}(G M)^{-1 / 7} 2^{-3 / 7}$.

Replacing the magnetic moment $\mu$ with the surface magnetic field strength $B_{\mathrm{NS}}=\mu / R_{\mathrm{NS}}^{3}$ at the magnetospheric equator with the neutron star radius $R_{\mathrm{NS}}$ and substituting the luminosity $L=$ $\dot{M} G M / R_{\mathrm{NS}}$, we obtain

$r_{\mathrm{m}}=B_{\mathrm{NS}}^{4 / 7} R_{\mathrm{NS}}^{12 / 7}(G M)^{-1 / 7} 2^{-3 / 7}\left(\frac{L R_{\mathrm{NS}}}{G M}\right)^{-2 / 7}$.
The correct inner radius $r_{0}$ of the disk depends on how the magnetic field threads the disk, which in turn depends strongly on the assumed accretion flow (see, e.g., Wang 1996, and references therein). Here we approximate this factor with a scalar factor $k$, with $k$ typically between $0.5-1$ :

$r_{0}=k \times r_{\mathrm{m}}$.

Replacing the variables with values of the expected order of magnitude, i.e., $L_{37}$ is the luminosity in $10^{37} \mathrm{erg} \mathrm{s}^{-1} \mathrm{~cm}^{-2}, B_{12}$ is the magnetic field in $10^{12} \mathrm{G}, M_{1.4}$ is the mass of the neutron star in $1.4 M_{\odot}$, and $R_{6}$ is the neutron star in $10^{6} \mathrm{~cm}$, we write

$r_{0}=3.0 \times 10^{8} \mathrm{k} B_{12}^{4 / 7} M_{1.4}^{1 / 7} L_{37}^{-2 / 7} R_{6}^{10 / 7} \mathrm{~cm}$.

On the other hand, the Keplerian co-rotation radius is defined by

$r_{\mathrm{c}}=(G M)^{1 / 3}\left(\frac{P}{2 \pi}\right)^{2 / 3}=1.7 \times 10^{8} P^{2 / 3} M_{1.4}^{1 / 3} \mathrm{~cm}$

with $P$ being the pulse period.

The onset of the propeller effect occurs at a luminosity $L_{\text {prop }}$, where $r_{\mathrm{m}}=r_{\mathrm{c}}$ and thus

$1.7 \times 10^{8} P^{2 / 3} M_{1.4}^{1 / 3} \mathrm{~cm}=3.0 \times 10^{8} k B_{12}^{4 / 7} M_{1.4}^{1 / 7} L_{\text {prop }}^{-2 / 7} R_{6}^{10 / 7} \mathrm{~cm}$,

which finally gives us

$L_{\text {prop }}=7.3 \times k^{7 / 2} P^{-7 / 3} R_{6}^{5} B_{12}^{2} M_{1.4}^{-2 / 3}$

in units of $10^{37} \mathrm{erg} \mathrm{s}^{-1}$.

\footnotetext{
4 Assuming a cross section of $4 \pi$ and neglecting a possible reduction by a factor $\xi$ for non-spherical accretion (Lamb et al. 1973).
} 